3 National Institute for Health and Clinical Excellence. Depression in Adults: The Treatment and Management of Depression in Adults (Clinical Guideline 90). National Institute for Health and Clinical Excellence, 2009

4 Fransen G, van Marrewijk C, Mujakovic S, Muris J, Laheij R, Numans M, et al. Pragmatic trials in primary care. Methodological challenges and solutions demonstrated by the DIAMOND-study. BMC Med Res Method 2007; 7: 16.

5 Even C, Siobud-Dorocant E, Dardennes RM. Critical approach to antidepressant trials. Blindness protection is necessary, feasible and measurable. Br J Psychiatry 2000; 177: 47-51.

6 Sullivan G, Oquendo M, Milak M, Miller J, Burke A, Ogden R, et al. Positron emission tomography quantification of serotonin $1 \mathrm{~A}$ receptor binding in suicide attempters with major depressive disorder. JAMA Psychiatry 2015; 72: $169-78$.

Bryony R. Corbyn, Psychiatry CT3, Mukesh Kripalani, London Deanery.

Email: Bryony.Corbyn@nhs.net

doi: 10.1192/bjp.206.5.434a

Author's reply: Dr Corbyn and Dr Kripalani's statement that our report 'brings into question the pharmacological advantage of antidepressant medication over placebo' is not warranted because our study was designed only to elucidate factors contributing to the placebo response in clinical trials. High placebo response rates in major depressive disorder (MDD) commonly lead to 'failed' trials (i.e. no statistical difference between drug and placebo). ${ }^{1}$ The fact that the medications showed numerical but not statistically significantly greater efficacy than placebo therefore is not surprising. Corbyn \& Kripalani suggest that the lack of statistical difference could represent a type II error. They are correct that we had limited power to detect such a difference, but this is not an error per se because the study was neither designed nor powered to examine the question.

Prior work has suggested that medication might not offer greater benefits than placebo except in moderate to severe depression. ${ }^{2}$ Corbyn \& Kripalani question whether the symptom severity in our sample was adequate to test our hypotheses. They specifically question our use of the HRSD, which they describe as 'irretrievably flawed', and ask whether they 'can be assured that participants met criteria for at least moderate depressive symptoms. First, as stated above, our aim was not to compare the efficacy of medication and placebo, so this concern is not relevant to the conclusions of our report. Second, all participants had diagnoses of MDD established using a structured interview instrument (Mini-International Neuropsychiatric Interview). Third, while there is no perfect symptom rating scale, the HRSD is the most widely used in clinical trials and does have some advantages over other instruments. ${ }^{3}$ The required score of $>17$ ensured that all participants met a commonly used threshold for depression treatment trials.

Corbyn \& Kripalani also ask for clarification regarding our choice of sample size. The study was powered to test our primary hypotheses, and the adequacy of the sample size can be assessed in part through the effect sizes of the regression analyses presented in Table 3 (p. 447). Our analyses examining expectations as predictors of outcome yielded highly significant results.

Corbyn \& Kripalani also express concern that 'recruitment via advertisement can be prone to selection bias and account for loss of external validity within studies'. All recruitment methods may introduce selection bias by including only a subset of those with MDD. For example, recruiting participants from a clinic biases a sample towards those who are better equipped to seek conventional care and who want only bona fide medication treatment, as opposed to those who may face barriers in accessing a clinic and are willing to possibly receive placebo in a research study. Because advertising for participants is a widely employed method for treatment research in MDD, our findings are likely to be relevant to other treatment study populations.

Additionally, Corbyn \& Kriplani's raise questions about the effectiveness of the treatment blinding in this study. We cannot determine whether there was any interaction between treatment assignment and nurses' symptom ratings. It is important to note, however, that raters remained blinded to the primary measure of interest in our results (expectation of the effectiveness of medications). Because these were formed at baseline, there was no possible influence of the nurses on this measure. Furthermore, as Corbyn \& Kripalani point out, there was no significant difference in depression treatment outcomes between medication and placebo treatment. It therefore seems unlikely that imperfections in the blinding would have been a significant contributor to our results. They also question whether 'suicidal behaviour' may have confounded our study results. Participants with any significant suicidal ideation were excluded from this study because of the possibility of placebo treatment.

Our report identified a novel form of expectation that contributed to heterogeneity in response to placebo. Corbyn \& Kripalani's letter highlights the fact that the design of the clinical trial itself also may contribute to heterogeneity in outcome. Their analysis underscores the need for future studies to examine the role of expectations in placebo response to confirm our results.

1 Leuchter AF, Hunter AM, Tartter M, Cook IA. Role of pill-taking, expectation and therapeutic alliance in the placebo response in clinical trials for major depression. Br J Psychiatry 2014; 205: 443-9.

2 Fournier JC, DeRubeis RJ, Hollon SD, Dimidjian S, Amsterdam JD, Shelton RC, et al. Antidepressant drug effects and depression severity: a patient-level meta-analysis. JAMA 2010; 303: 47-53.

3 Bech P. Rating scales in depression: limitations and pitfalls. Dialogues Clin Neurosci 2006; 8: 207-15.

Andrew F. Leuchter, MD, Aimee M. Hunter, PhD, Molly Tartter, PhD, Ian A. Cook, MD, University of California - Los Angeles. Email: afl@ucla.edu

doi: 10.1192/bjp.206.5.435

\section{Improving assessment and treatment of physical health problems in people with severe mental illness: the case for a shared IT system}

The poor assessment and treatment of physical health problems in people with schizophrenia found by Crawford et al is sadly not surprising.

The methodological problems that became evident in the pilot phase mirrors the problems faced in practice - many trusts do not have up-to-date physical health monitoring and these must be requested from primary care. Clinicians in mental health services interested in getting this information and who want to actively take part in physical health assessment and treatment have to, like Crawford et al, write to the general practitioner (GP) requesting this information and hope for a timely response.

Out of hours, there is no simple way of checking current medication, physical health conditions and allergies: information that is readily available on primary care databases. Many clinicians in mental health spend considerable time contacting GP practices to request investigations and results. Conversely, GPs are often frustrated at not finding out about changes in management plans and psychotropic medication quickly enough. This system of care is not conducive to the urgent need to improve physical healthcare for this group of patients.

A shared IT platform for primary care and mental health services, with up-to-date information on physical health such as 